\title{
PROJETO DEMONSTRATIVO EM EPILEPSIA NO BRASIL
}

\author{
Li Li Min', J.W.A.S. Sander
}

\begin{abstract}
RESUMO - A epilepsia é a condição neurológica grave de maior prevalência no mundo. Infelizmente, a maioria dos pacientes com epilepsia nos países em desenvolvimento não recebem tratamento adequado e sofrem estigmatização pelo diagnóstico. Diante deste cenário, em 1997 foi lançada uma Campanha Global Contra Epilepsia liderada pela Organização Mundial da Saúde, a Internacional League Against Epilepsy, e International Bureau for Epilepsy. A Campanha entrou na sua segunda fase em 2001 com os projetos demonstrativos que estão em curso na China, Zimbábue, Senegal e recentemente no Brasil. A finalidade do projeto demonstrativo é mostrar através de avaliação metodológica que é possível estabelecer um modelo de atendimento integral aos pacientes com epilepsia. O projeto demonstrativo brasileiro tem como regiões de atuação Campinas e São José de Rio Preto. Uma Task Force foi instituída para estudar a expansão da atuação para outras áreas do país.
\end{abstract}

PALAVRAS-CHAVE: epilepsia, estigma, prevenção, tratamento, drogas antiepiléticas.

\begin{abstract}
National demonstration project on epilepsy in Brazil
ABSTRACT - Epilepsy is the most prevalent non-communicable serious neurological condition worldwide. Unfortunately, the majority of people with epilepsy in low-income countries do not receive appropriate treatment. Stigmatisation is the rule. In this setting, the World Health Organisation, the International League against Epilepsy and the International Bureau for Epilepsy launched the Global Campaign against Epilepsy in 1997. This entered its second phase in 2001 and as part of it has set up demonstration projects in the People's Republic of China, Zimbabwe, Senegal and, more recently, in Brazil. The objective of the demonstration projects is to show, through methodological evaluation, that it is possible to establish a model of treatment for people with epilepsy in primary health care settings. The Brazilian demonstration project has targeted regions in Campinas and São Jose do Rio Preto, both in Sao Paulo State. A task force has been established to assess strategies to expand this project nationwide.
\end{abstract}

KEY WORDS: epilepsy, stigma, prevention, treatment, antiepileptic drug.

\section{CAMPANHA GLOBAL EPILEPSIA} FORA DAS SOMBRAS NO BRASIL

A epilepsia é a condição neurológica grave de maior prevalência no mundo. Ela acomete $1 \%$ da população, ou seja 60 milhões de pessoas no mundo e a cada ano somam-se aproximadamente três milhões de casos novos ${ }^{1}$. Pelo menos $50 \%$ dos casos começam na infância ou adolescência.

Epilepsia certamente não é um fenômeno recente como mostram documentos originários do oriente há mais de 4 mil anos. $O$ tratamento medicamentoso eficaz (brometo) está disponível há mais de 100

\footnotetext{
${ }^{1} \mathrm{MD}, \mathrm{PhD}$, Professor Doutor do Departamento de Neurologia da Universidade Estadual de Campinas, Campinas SP, Brasil (UNICAMP), representando o Projeto ASPE (Assistência à Saúde de Pacientes com Epilepsia) executor da Campanha Global Contra Epilepsia no Brasil; ${ }^{2} \mathrm{MD}, \mathrm{PhD}$, Professor de Neurologia da National Hospital of Neurology and Neurosurgery - Queen Square e Líder Científico da Campanha Global Contra Epilepsia, representando o Comitê Internacional da Campanha Global Contra Epilepsia - "Driving Epilepsy from out of the Shadows" coordernado pela Organização Mundial da Saúde, International League Against Epilepsy e International Bureau of Epilepsy. Fazem parte do comitê executivo do projeto ASPE: Carlos Alberto Mantovani Guerreiro. Professor Associado do Departamento de Neurologia da UNICAMP; Marilisa Mantovani Guerreiro, Professora Associada e Chefe do Departamento de Neurologia da UNICAMP; Fernando Cendes, Professor Doutor do Departamento de Neurologia da UNICAMP, Chefe do Laboratório de Neuroimagem da UNICAMP; Ana Lúcia Andrade Noronha, Neurologista; Lucia Helena Marques, Professora Assistente do Departamento de Neurologia da Faculdade de Medicina de São José de Rio Preto, São José do Rio Preto SP, Brasil (FAMERP); Moacir Borges, Professor Assistente do Departamento de Neurologia da FAMERP; Elisabete Abib Pedroso de Souza, Professora Doutora do Departamento de Neurologia da UNICAMP e Chefe do Laboratório de Pesquisa em Qualidade de Vida da UNICAMP; Paula Teixeira Fernandes, Psicóloga; Fernanda Doretto, Psicóloga; Priscila Salgado, Psicóloga; Carlos Vogt, Diretor do Laboratório de Jornalismo da UNICAMP; Leonardo Bonilha, Estudante de doutorado; Pablo Rio, Gerente de sistema; Ulisses de Araújo, Professor Doutor da Faculdade de Educação da UNICAMP; Maria Tereza Eglér Mantoan, Professora Doutora Chefe do Laboratório de Pesquisa em Diversidade e Educação da Faculdade de Educação da UNICAMP; Dirce Maria Zanetta, Professora Doutora e Chefe do Departamento de Epidemiologia e Saúde Pública da FAMERP; Raul Aragão Martins, Professor Doutor da Faculdade de Educação da UNESP; José Cacau Lopes, Secretário de Saúde de São José do Rio Preto e Gastão Wagner de Souza Campos, Secretário de Saúde de Campinas.
}

Recebido 5 Dezembro 2002. Aceito 16 Dezembro 2002.

Dr. Li Li Min - Departamento de Neurologia - FCM - UNICAMP - Caixa Postal 6111 - 13083-970 Campinas SP - Brasil. FAX: $193788-7483$.

E-mail: limin@fcm.unicamp.br (http://www.aspe.hc.unicamp.br) 
anos e as crises podem ser controladas na sua grande maioria (70\%) com medicação de baixo custo, propiciando assim uma vida normal a estes pacientes. Para aqueles que não respondem ao tratamento medicamentoso, há possibilidade de tratamento cirúrgico, disponível há mais de 100 anos. Pelos relatos hipocráticos ${ }^{2}$ de mais de 2,4 mil anos, sabemos que epilepsia não é uma condição de causa demoníaca ou espiritual, e os avanços científicos na área são contínuos e constantes, com um número expressivo de recentes publicações em literatura internacional (>66 mil publicações no Banco do PubMed usando palavra epilepsy).

Contudo, somente $10 \%$ a $40 \%$ dos pacientes recebem tratamento medicamentoso ${ }^{3}$ e a oferta de tratamento cirúrgico é praticamente inexistente. Os médicos, incluindo uma parcela de neurologistas, não estão preparados para atender pacientes com epilepsia. O sistema de saúde primário no manejo de pacientes com epilepsia é inexistente. Sabe-se que o não-tratamento está associado a maior morbidade e risco de morte súbita ${ }^{4}$, que provavelmente é maior nos países em desenvolvimento, como o Brasil. $\mathrm{O}$ encargo sócio-econômico da epilepsia ativa é desconhecido, mas provavelmente muito alto no Brasil.

As crenças e mitos que envolvem a epilepsia parecem ser a regra na comunidade. Muitos ainda acreditam em posseção demoníaca como a explicação para epilepsia e buscam os tratamentos afins que incluem o exorcismo e simpatias. Esta ignorância tem contribuído na perpetuação da estigmatização, uma triste realidade que desclassifica e exclui os indivíduos com epilepsia e seus familiares da sociedade.

Tendo em vista esta situação crítica dos pacientes com epilepsia no mundo, uma campanha global liderada pela Organização Mundial da Saúde, Liga Internacional Contra Epilepsia (ILAE) e Organização Internacional dos Pacientes com Epilepsia (IBE), foi lançada em $1997^{5}$ em alguns países da África com intuito principal de fornecer esclarecimento e desmi-

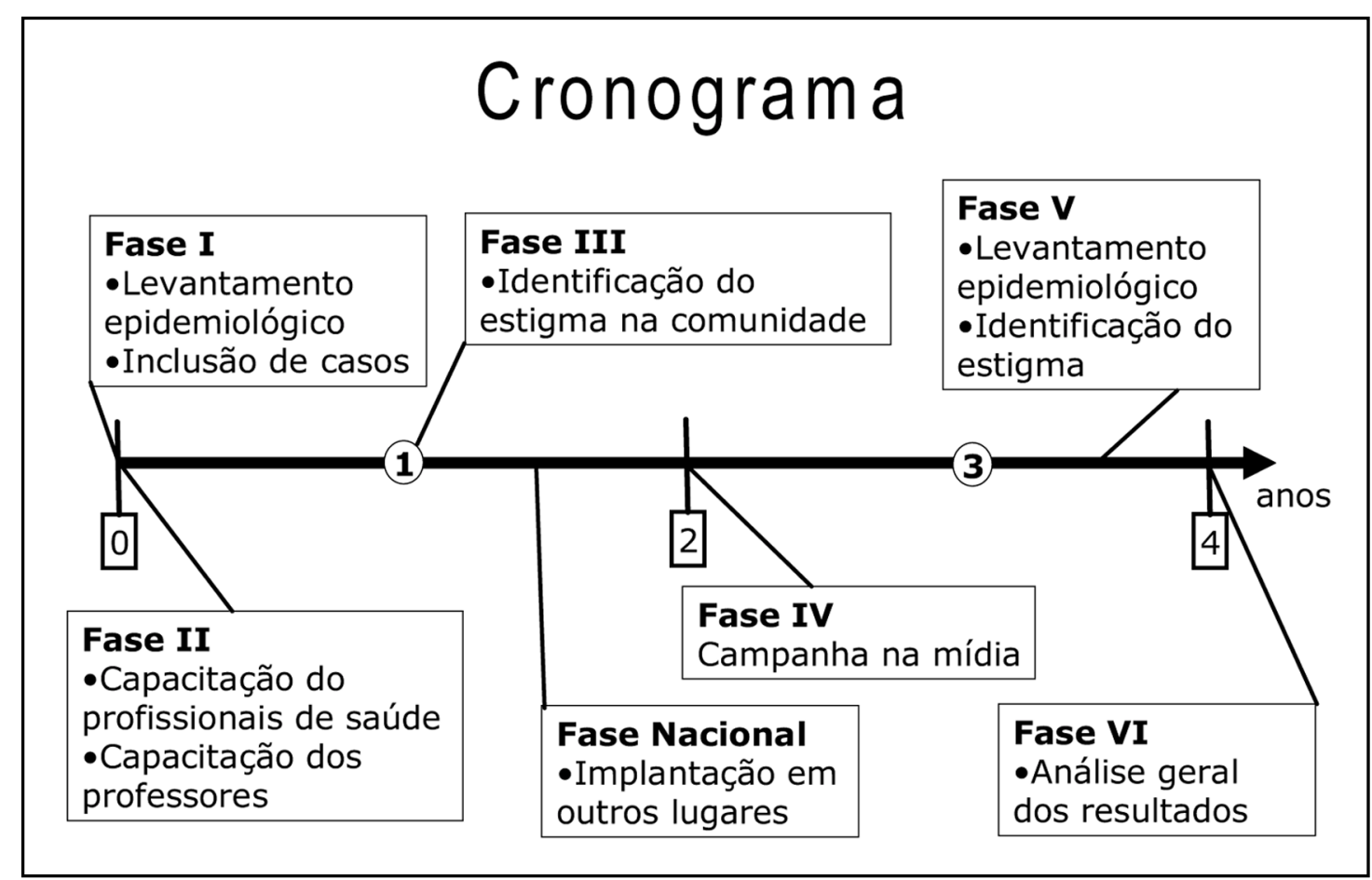

Figura. O projeto demonstrativo tem a duração de quatro anos (linha horizontal representa o tempo) e é constituído de seis fases principais (os retângulos contêm as principais atividades de cada fase). A Fase I consiste de com rastreamento epidemiológico porta-a-porta utilizando um questionário estruturado visando definir a população a ser estudada em duas comunidades de aproximadamente 100 mil habitantes. A Fase II consiste em cursos de capacitação de profissionais da rede básica de saúde usando módulo de treinamento teórico-prático de 8 horas de duração. Este curso de capacitação está sendo incooporado no programa médico de família em Campinas e São José do Rio Preto. A capacitação de professores das escolas de ensino fundamental é realizada através de um curso de extensão - Formação ética, temas transversais epilepsia, com duração de 38 horas. A Fase III é o estudo epidemiológico para definir os fatores operantes na perpetuação do estigma na comunidade e a Fase IV é campanhas de intervenção usando a mídia. As fases V e VI são a avaliação epidemiológica para estimar o grau de mudação obtidas com as intervenções e análise dos reusltados. A Fase Nacional é coordenada pela Task Force, concomitante ao projeto ASPE, para expandir as experiências para outras localidades do país. 
tificação da Epilepsia. Em 2001, a Campanha Global entrou na sua segunda fase com ações centradas nos projetos demonstrativos. Os projetos demonstrativos têm como ponto principal o aspecto intervencionista, de forma a promover modificação no sistema de saúde, otimizando tratamento, diagnóstico e prevenção da epilepsia na comunidade, visando em última análise, fornecer qualidade de vida melhor a estes indivíduos.

$\mathrm{O}$ presente projeto demonstrativo brasileiro foi submetido, analisado e aprovado por um comitê internacional composto por membros de cada uma das organizações internacionais responsáveis pela Campanha Global. A partir do dia 31 de junho de 2002 o projeto ASPE (Assistência à Saúde de Pacientes com Epilepsia) foi aceito como integrante oficial da campanha global. Existem quatro projetos demonstrativos no mundo: China, Zimbabwe, Senegal e agora Brasil. Uma reunião de trabalho e assessoria aberta com o comitê internacional foi realizada com intuito de definir o cronograma das fases do projeto ${ }^{6}$ (ver Figura). $\mathrm{O}$ início oficial do projeto demonstrativo brasileiro ocorreu dia 23 de setembro de 2002.

O intuito deste artigo é 1) levar ao conhecimento da comunidade neurológica brasileira a natureza deste projeto, os objetivos e a metodologia empregada. 2) formular junto com os colegas ações que possam ser multiplicadas a outras regiões no Brasil mediante um esforço coletivo e em diferentes frentes, com o objetivo de proporcionar um incremento na qualidade de vida dos pacientes com epilepsia e seus familiares. A mudança desta situação paradoxal só ocorrerá na medida em que todos entendam que a epilepsia é um problema de saúde pública. A solução requer uma resposta social e médico-assistencial ampla e mantida. Tal ação está amparada pelo fato de que a epilepsia é uma condição tratável.

\section{OBJETIVOS}

\section{Os objetivos gerais do projeto são:}

- Avaliar procedimentos que melhorem a identificação e o manejo de pessoas com epilepsia na área urbana através do sistema de atendimento primário à saúde já existente e com a participação da comunidade.

- Desenvolver um modelo de tratamento integral de epilepsia que possa ser aplicado em âmbito nacional, tendo como palco principal a rede primária de saúde.

\section{Especificamente, o projeto demonstrativo visa:}

1) Avaliar a prática de manejo corrente (identificação, tratamento e seguimento) de pacientes com epilepsia na área urbana do país.

2) Estimar: a) a prevalência das formas ativas de epilepsia;

b) a porcentagem da lacuna de tratamento via uma metodologia ativa de localização de casos; c) as mudanças que o projeto pode trazer na área de estudo.

3) Determinar a etiologia e fatores de risco em associação com a epilepsia na comunidade.

4) Reduzir e erradicar causas preveníveis de epilepsia na comunidade.

5) Determinar o conhecimento, as atitudes e o atendimento de pacientes com epilepsia entre os profissionais de saúde na rede primária de saúde antes e depois deles terem sido submetidos a um treinamento em epilepsia.

6) Desenvolver normas técnicas para identificação, educação, tratamento e seguimento de pacientes com epilepsia na rede primária de saúde.

7) Promover um estudo de tratamento de formas de epilepsia, usando antiepilépticos de primeira linha pelos médicos do atendimento primário à saúde.

8) Desenvolver estratégias para a implementação de um programa cirúrgico custo-efetivo para o tratamento de epilepsias refratárias a medicações antiepilépticas.

9) Desenvolver um programa de educação profissional continuada em epilepsia para profissionais da rede primária de saúde.

10) Promover consciência pública sobre epilepsia via um programa educacional direcionado à comunidade.

11) Promover educação continuada para professores de escolas primárias e secundárias e difusão de informações sobre epilepsia.

12) Desenvolver um programa de desestigmatização da epilepsia e de melhora da aceitação social.

13) Promover grupos de gerenciamento e suporte para pessoas com epilepsia.

14) Reduzir a carga econômica e social da epilepsia nas áreas estudadas.

\section{METODOLOGIA}

O projeto tem como metodologia a pesquisa operacional com ênfase no acompanhamento da qualidade e dos resultados (eficácia e eficiência) e pode ser dividida em três partes:

- Estimativa Epidemiológica. Esta parte tem como finalidade fornecer uma estimativa real da prevalência da epilepsia e da epilepsia ativa não tratada na área estudada bem como o grau de estigma e aceitação social.

- Estudo de Intervenção. Esta parte visa desenvolver melhorias nas questões de diagnóstico, tratamento com drogas anti-epiléticas (DAEs), seguimento e rede de referências. 
- Intervenção Educacional, Social e Comunitária. Esta parte compreende promoção dos aspectos educacional e social do projeto.

\section{COMITÊ EXECUTIVO DO}

\section{PROJETO DEMONSTRATIVO}

O Comitê Executivo está sediado na Universidade Estadual de Campinas (UNICAMP), sob a coordenação de Li Li Min (UNICAMP) e Ley Sander (University College London). Duas subcomissões: tratamento e desestigmatização foram criadas para otimizar o desenvolvimento destas duas importantes áreas. A subcomissão de tratamento é liderada por Carlos Alberto Mantovani Guerreiro e a subcomissão de desestigmatização é liderada por Elisabete Pedroso Abib de Souza. Marilisa Guerreiro proverá consultoria em epilepsia em crianças. Além disso, Fernando Cendes estudará alternativas de implementação de um programa cirúrgico custo-efetivo para o tratamento de pacientes com epilepsia refratária à medicação. Lúcia Marques e Moacir Alves Borges da Faculdade de Medicina de São José do Rio Preto (FAMERP) serão os facilitadores regionais em São José do Rio Preto.

Este projeto será aplicado no Distrito de Barão Geraldo, em Campinas, e bairros Santo Antônio e Jaguaré, em São José do Rio Preto. A população total destas áreas totaliza aproximadamente 100 mil habitantes. A principal área de atuação deste projeto estará centrada nos centros de saúde de atenção primária e seus centros terciários de referência (Hospital Universitário da UNICAMP e da FAMERP).

\section{MENSURAÇÕES DE RESULTADOS}

Uma série de avaliações será realizada para medir a efetividade do projeto. As aferições de resultados são baseadas no guia apresentado durante o Encontro sobre o Impacto da Epilepsia.

Um dos principais pontos de avaliação da eficácia do projeto é a questão do tratamento.

1. Toda a eficácia do projeto em reduzir a lacuna de tratamento será determinada por duas estimativas epidemiológicas da lacuna de tratamento que serão levantadas no início do estudo e quatro anos mais tarde, por mensurações de mudanças no número de pacientes não tratados entre os dois pontos de estimativa.

2. A eficácia do manejo de pessoas com epilepsia no centro de saúde primário será determinada por:

2.1. A eficácia clínica da intervenção (número de pacientes sem crises e com significativa melhora das crises);

2.2. Segurança e tolerabilidade do tratamento (número de pessoas que desistiram devido aos efeitos colaterais)

2.3. Mudanças sociais observadas em pacientes e na comunidade.

3. A eficácia do módulo de treinamento para induzir mudanças no conhecimento, na atitude e na prática entre os profissionais de saúde será mensurada por:

3.1. Mudanças na informação registrada nos questionários para avaliar o grau de conhecimento, atitude e percepção da epilepsia, a partir do começo do treinamento e 6 meses depois;

3.2. Mudanças no número de pacientes identificados e tratados pelos profissionais de saúde.

4. A eficácia do programa para reduzir o encargo social e econômico da epilepsia será mensurada por:

4.1. O número de pacientes que retornam à escola ou conseguem emprego; ou começam a trabaIhar ou a ir para a escola pela primeira vez;

Mudanças nos registros de freqüência (diminuição do absenteísmo) daqueles na escola ou no trabalho.

\section{TASK FORCE - AMPLIAÇÃO PARA REDE NACIONAL}

Visando ao aproveitamento dos resultados obtidos durante este projeto demonstrativo, uma Task Force foi formada e estudará estratégias para a expansão do modelo para todo o Brasil. Esta Task Force é coordenada pela Liga Brasileira de Epilepsia sob a liderança do seu presidente Américo Ceiki Sakamoto. Participam desta Task Force, Marly de Albuquerque (Presidenta da Associação Brasileira de Epilepsia), Carlos Eduardo Silvado (último presidente da LBE) e Li Li Min (coordenador do projeto ASPE). Para que as ações mudem a situação crítica dos pacientes com epilepsia é necessária a participação de todos, seja na formulação de propostas, críticas, sugestões. Encorajamos que procurem a Liga Brasileira de Epilepsia para envolver as suas localidades na área de abrangência desta ação para que possamos colocar a epilepsia sob nova perspectiva no Brasil.

\section{REFERÊNCIAS}

1. Sander JW, Shorvon SD. Epidemiology of the epilepsies. J Neurol Neurosurg Psychiatry 1996;61:433-443.

2. Temkin O. The Falling Sickness: a history of epilepsy from the Greeks to the beginnings of modern neurology. 2.Ed. Baltimore: John Hopkins Univ Press, 1994

3. Kale R. Global campaign against epilepsy:the treatment gap. Epilepsia 2002;43(Suppl 6):31-33

4. Nashef L, Fish DR, Sander JW, Shorvon SD. Incidence of sudden unexpected death in an adult outpatient cohort with epilepsy at a tertiary referral centre. J Neurol Neurosurg Psychiatry 1995; 58:462-464.

5. Sander JW. Global Campaign against epilepsy: overview of the demonstration projects. Epilepsia 2002;43(Suppl 6):34-36.

6. Kobayashi E, Li LM, Sander JW. Report of Brazilian Demonstration Project. http://www.aspe.hc.unicamp.br/pt/pdf/Report_Demo_ Project_Brazil_Workshop.pdf .17-8-2002. 